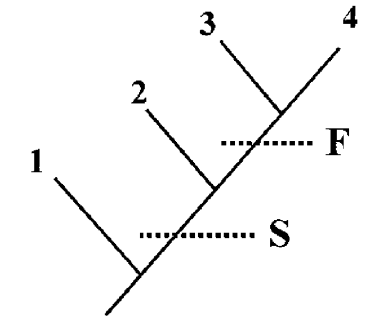

A.

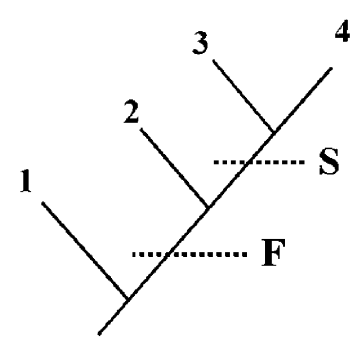

B.

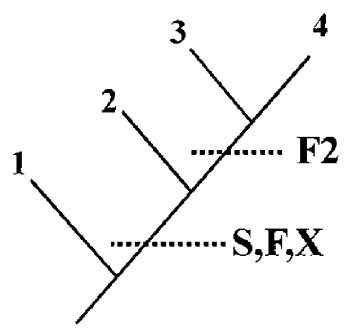

C.

D.

Figure 1 (Blackburn). Cladograms of four taxa, showing the timing of evolution of a given structure ("S") and function ("F"), as inferred from characteristics of the taxa. In A, the structure evolutionarily predates a function that it serves, indicating exaptation. In $\mathrm{B}$ and $\mathrm{C}$, the function respectively predates or accompanies evolution of the structure; such sequences are necessary (but not entirely sufficient) indicators of adaptation. In D, a spandrel (X) has accompanied evolution of an adapted structure (S) as an architectural byproduct; here, the structure subsequently is exapted for a new function (F2).

guishing exaptation from adaptation by determining whether character traits have predated, accompanied, or followed evolution of their functional attributes (e.g., see Blackburn 2000; Larsen \& Losos 1996). The enclosed figures illustrate acladograms of hypothetical taxa, with various possible phylogenetic distributions of a particular structure (or other phenotypic characteristic) and a function superimposed. The timing of evolution of a feature is inferred from its taxonomic distribution (Brooks \& McLennan 1991). Thus, in Figure 1A, the presence of a derived structure ("S") in three of the taxa indicates that it probably characterized their common ancestor. When the structure originates first and only later takes on the function in question (as in the mammary example above), exaptation is indicated (Fig. 1A). When a function either predates (Fig. 1B) or accompanies (Fig. 1C) evolution of a structural feature, it may represent a case of adaptation. Phylogenetic analysis also may facilitate recognition of spandrels. An architectural byproduct should originate as an unselected correlate of a particular structure, regardless of whether the structure itself is selected (Fig. 1D).

In phylogenetic analysis, adaptation and exaptation have the status of mutually exclusive, competing hypotheses, each of which can be falsified or supported according to the sequence of evolutionary modification. That a structure antedated the function it performs, offers a sufficient criterion for recognition of exaptation. However, a function predating or accompanying evolution of a given structure is a necessary but insufficient criterion for recognition of adaptation; therefore, other criteria (such as those discussed by Andrews et al.) must also be brought to bear. In effect, phylogenetic analysis allows us to address, in an evolutionary context, difficult issues whose recognition dates to the writings of David Hume - notably the difficulty of distinguishing causation from correlation.

As a practical matter, phylogenetic analysis is useful chiefly where a robust cladogram can be constructed from taxa that vary in structural and functional features of interest. It therefore offers no panacea to evolutionary psychology, where inferences of genetically based behavioral attributes are problematic, particularly as applied to extinct hominids. However, in principle, phylogenetic approaches offer ways to analyze evolutionary sequences and transformations in historical contexts, and where sufficient data are available, they can provide clear evidentiary standards for distinguishing exaptation from adaptation.

ACKNOWLEDGMENT

I thank Kent Dunlap for reviewing a draft of this commentary.

\section{There is no evidentiary silver bullet for the frequency adaptation hypothesis}

\author{
Gary L. Brase \\ Division of Psychology, Sunderland Business School, University of \\ Sunderland, Sunderland, SR6 ODD United Kingdom. \\ gary.brase@sunderland.ac.uk \\ http://www.sunderland.ac.uk/ bs0gba
}

Abstract: Special design criteria are largely unable to discriminate between claims that specific competencies in judgements under uncertainty are a result of an adaptation for representing naturally sampled frequencies, or due only to inherent properties of such a format. Because divisions between these perspectives are thin, evidence via additional criteria are persuasive only in combination, using inference to the best available explanation.

Andrews et al. point out, quite correctly, that different traits may require satisfaction of different evidentiary criteria in reaching some consensus on whether it is an adaptation. This may actually not be a strong enough statement of the case: In many situations, one or more of the usual evidentiary criteria may be used to argue against the case for adaptation. A case in point is the recent debates on the nature of statistical judgements under uncertainty.

The claim has been made that information in the form of frequencies, and in particular frequencies in a natural sampling framework, is privileged representational format (i.e., that it is the proper domain for a cognitive adaptation for making statistical judgements; Cosmides \& Tooby 1996; Gigerenzer \& Hoffrage 1995). Those in opposition to this claim have pointed out that naturally sampled frequencies create computationally less complicated situations simply by virtue of their inherent properties and they reject the claim of a specific adaptation (e.g., Evans et al. 2000; Girotto \& Gonzalez 2001; Johnson-Laird et al. 1999). In particular, the counter-hypothesis to an adaptationist explanation is that, because the frequencies within a natural sampling system inherently preserve base-rate information, the set/subset relationships between classes of events become much more easily perceived (see Fig. 1). Instead of an adaptation for understanding and using frequencies, this explanation rests on claims for a basic appreciation of set relations (which happen to be expressible almost exclusively in frequentist terms).

In the context of this debate, criteria such as proficiency, efficiency, economy, and reliable production are unable to discriminate between these two explanations; and in fact, the presence of these features - attributed to the nature of the inputs (natural frequencies per se) - have been used to argue against an adaptationist interpretation. One can argue that the proficiency, efficiency, and economy of the behavior when using natural frequencies is purely the result of the properties of the numbers themselves (natural frequencies are simply easier), or one can argue that these characteristics are a result of a cognitive mechanism that is preferentially tuned to using these numerical formats in the first place (natural frequencies are particularly easy because the mind is designed to work with them).

Discriminating between these two theoretical perspectives is difficult because the divisions between them have become thin, 


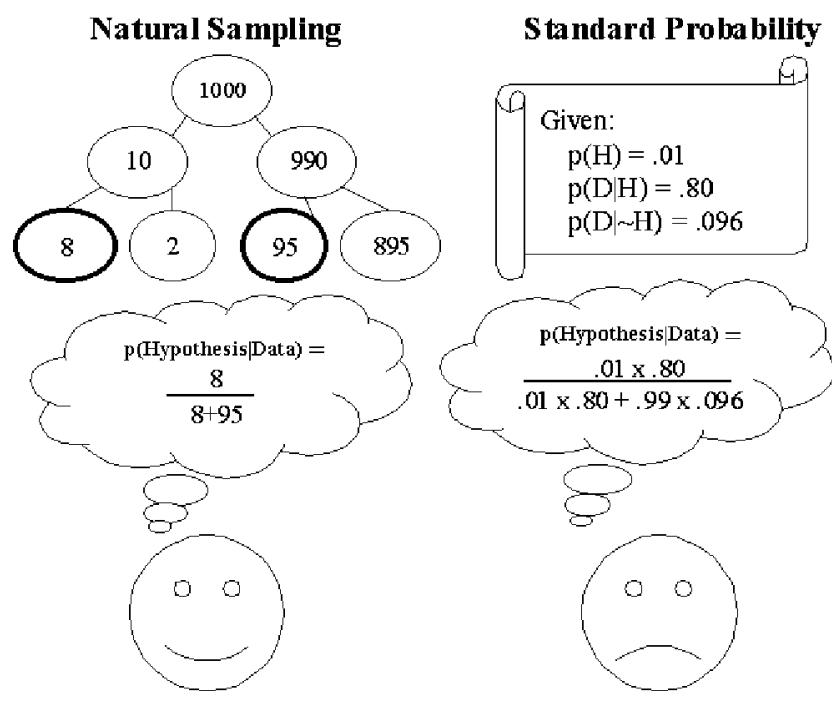

Figure 1 (Brase). Natural sampling versus standard probability. Differences in computational complexity in calculating the posterior probability (Bayesian inference), attributable to the information representation (natural sampling of frequencies versus singleevent probabilities). (Figure adapted from Gigerenzer \& Hoffrage 1995.)

and there is significant overlap in their predictions. Given this state, particularly, clear thinking about multiple, alternative evidentiary criteria is increasingly important. What evidentiary criteria can be used, then, in evaluating the adaptationist hypothesis that natural frequencies not only are computationally simpler (a point all parties agree on), but also constitute a privileged representational format?

Using the criteria of biased learning outcomes of developmental learning mechanisms would appear to clearly support the adaptationist view of frequencies. Children learn whole numbers (frequencies) relatively quickly and easily, but they often develop any of a number of characteristic difficulties when learning mathematical concepts that deviate from a frequentist perspective (Geary 1995; Geary \& Lin 1998). For example, children often develop misconceptions about the nature of fractions and decimals, and a large number of these misconceptions are recognizable as misapplications of a frequentist interpretation of numbers (Brase 2002a). Because numbers and mathematics are an academic topic, however, the possible confounding influences of teaching techniques can be raised as a concern (e.g., Glassman 1996). It is unclear whether an early proficiency with frequencies is a result of the evolved structure of the mind or of the exposure to frequency information and subsequent learning to use such information.

The criteria of specificity may need to be revised to become even more precise, such that it again discriminates between predictions made based on different hypotheses. For example, based on the notion that an adaptation for tracking natural frequencies of objects, events, and locations in the real world must have some set of parsing rules for dividing the world into countable entities, Brase et al. (1998) proposed that such an adaptation should operate better on whole objects, events, and locations than on arbitrary aspects or features of such entities (i.e., the individuation hypothesis). Their subsequent experiments documented that, indeed, people's statistical judgements were hampered in tasks that required calculations about aspects of objects and the objects themselves, even if the information was presented in natural frequencies. Statistical judgements about individuated objects were consistently more successful.

Criteria such as fit with the ancestral environment, rather than the modern world, are difficult to assess because statistically in- formed judgements in ancestral circumstances would be translated directly into behavior without necessarily any explicit and conscious mathematical calculations. Although we know that bumblebees perform complex calculations of posterior probabilities, we know little about the performance of human hunter-gatherers in this respect. Related research, however, appears to support the idea of statistical judgement abilities adapted to an ancestral world. Isomorphic numerical information, presented in different formats, is evaluated and perceived in very different ways, and these phenomena can help in understanding the ways that such information is cognitively represented (Brase 2002b; Wang 1996). Specifically, some irrational and inconsistent responses in the face of statistical judgements become apparent only when using numbers on a scale that would never have been encountered at any time in evolutionary history (e.g., dealing with millions of people).

Ultimately, the adjudication about a trait being an adaptation must be made as an inference to the best available explanation, given all the evidence (preferably using multiple, independent, and converging lines of evidence). As troublesome as it may be, this means that weaker explanations can continue to survive in some quarters by selective attention to various lines of evidence (for example, by ignoring most of the information from outside one's own discipline). In this respect, one of the key obstacles to adaptationist descriptions of cognitive and behavioral traits is the narrowing of individual interests and knowledge within traditional academic divisions.

\section{ACKNOWLEDGMENTS}

The author thanks David Over and Sandra Brase for helpful comments and advice in the development of this comment.

\section{Development: The missing link between exaptationist and adaptationist accounts of organismal design}

\section{William Michael Brown}

Department of Psychology, Dalhousie University, Halifax, Nova Scotia, B3H 4J1, Canada.wmbrown@dal.ca http://www.dal.ca/ esg/WilliamMBrown.htm

Abstract: To understand adaptation (and exaptation), a more comprehensive view of development is required: one beyond a constraining force. Developmental plasticity may be an adaptation by natural selection simultaneously favored (or sometimes in conflict) at multiple levels of biological organization (e.g., cells, individuals, groups, etc.). To understand the interrelationships between developmental plasticity and adaptive evolution I borrow heavily from West-Eberhard (2003) and Frank (1995; 1997). Developmental plasticity facilitates evolution, results in particular patterns of evolutionary change, and may produce exaptations by design rather than by chance.

Development is often viewed as a source of exaptation in nature. However, a comprehensive theory of adaptive evolution must feature development beyond a constraining force. Another way of elucidating apparent exaptations is the concept of "phenotypic accommodation" (West-Eberhard 2003). Phenotypic accommodation is the nongenetic adjustment among interacting and variable-evolved components attributable to phenotypic plasticity (West-Eberhard 2003). West-Eberhard's view of development and evolution incorporates the latest findings in the evolutionary study of behaviour, genetics, endocrinology, and molecular mechanisms. Indeed, terms like exaptation (used in isolation from the adaptationist programme) and developmental constraints may have impeded synthesis between evolutionary and developmental biology.

Developmental plasticity causes phenotypic variation, which in turn is screened by selection. Mutations must first influence development to influence evolutionary change. Andrews et al. have discussed development mainly in terms of constraints (although, 